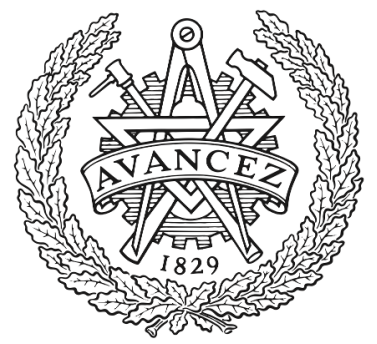

CHALMERS

UNIVERSITY OF TECHNOLOGY

\title{
PCB Based UWB mm-Wave Smart Capped Bowtie Array for 5G Communication Systems
}

Downloaded from: https://research.chalmers.se, 2023-04-26 12:31 UTC

Citation for the original published paper (version of record):

Yang, J., Mansouri Moghaddam, S., Uz Zaman, A. et al (2018). PCB Based UWB mm-Wave Smart Capped Bowtie Array for 5G Communication Systems. IEEE Antennas and Propagation Society International Symposium: 631-632. http://dx.doi.org/10.1109/APUSNCURSINRSM.2018.8609391

N.B. When citing this work, cite the original published paper. 


\title{
PCB Based UWB mm-Wave Smart Capped Bowtie Array for $5 \mathrm{G}$ Communication Systems
}

\author{
Jian Yang ${ }^{1}$, Sadegh Mansouri Moghaddam ${ }^{1}$, Ashraf Zaman ${ }^{1}$, Zhongxia Simon $\mathrm{He}^{2}$, Vessen Vassilev ${ }^{2}$ \\ ${ }^{1}$ Dept. of Electrical Enginering, ${ }^{2}$ Department of Microtechnology and Nanoscience \\ Chalmers University of Technology, Gothenburg, Sweden
}

\begin{abstract}
This paper gives an overview of the preliminary development of a new antenna technology - the UWB capped Bowtie array antenna aiming to the applications in 5G wireless communication systems. The capped Bowtie antenna has not only the ultra-wideband active reflection coefficient performance but also a UWB stable radiation functions of the array element. A novel solution to the aggregation for tightly coupled capped Bowtie array is also presented briefly in the paper.
\end{abstract}

\section{INTRODUCTION}

Demands on high data transfer rate over wireless systems increase every year. Smart millimeter-wave (mm-Wave) wireless systems are inevitable for high data rate communication such as $10-$ Gbps data transfer capacity in $5 \mathrm{G}$ communication systems. In 5G systems, the planned backhaul and fronthaul links may cover 15-43 GHz, E-band (71-86 GHz), W-band (92-115 GHz) and D-band (130-175 GHz), and the proposed mm-Wave access to users may have a band of $24-52 \mathrm{GHz}$ [1], [2]. In addition, 5G mm-Wave systems make antenna systems physically small, which opens up the possibility of implementation of electrically large massive MIMO array [3] at micro base stations and even inside mobile phones or other users devices such as cars and drones. One of the critical part, often a bottleneck, in developing mm-Wave smart wireless systems is the high-gain, low-cost, multibeam-steerable ultrawideband (UWB) array antennas. In this paper, we present a new antenna technology, UWB capped Bowtie array antenna aiming for applications to $5 \mathrm{G}$ systems.

\section{GeOMETRY OF CAPPED BowtiE ANTENNA}

Fig. 1 shows the simulation model and fabricated prototype of the capped Bowtie antenna [4] at microwave frequency, where the Bowtie arms and the cap are made of metal sheet supported by dielectric posts. Fig. 2 shows the simulated and measured reflection coefficient, which is below $-10 \mathrm{~dB}$ from 3 to $12 \mathrm{GHz}$, a 4:1 bandwidth. Simulated and measured radiation patterns in E- and H-planes at 4 frequency points are presented in Fig. 4. There are two unique radiation features in addition to the UWB performance: 1) $10 \mathrm{~dB}$ beamwidth is nearly constant and as broad as about $140^{\circ}$ over $3-12 \mathrm{GHz}$, even when the length of the Bowtie arms is about 2 wavelength $(\lambda)$ at 12 $\mathrm{GHz}$ (standard dipole or Bowtie has split beams for $2-\lambda$ long arms); 2) E- and H-plane radiation patterns are similar (a standard dipole has an Omni-direction pattern in H-plane and directive pattern in E-plane). These feature, due to the novel configuration of the capped Bowtie, are important for UWB

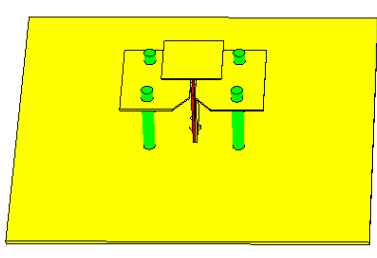

(a)

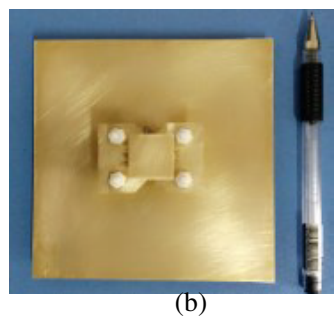

Fig. 1. Capped Bowtie antenna at microwave frequencies: (a) simulation model, and (b) prototype.

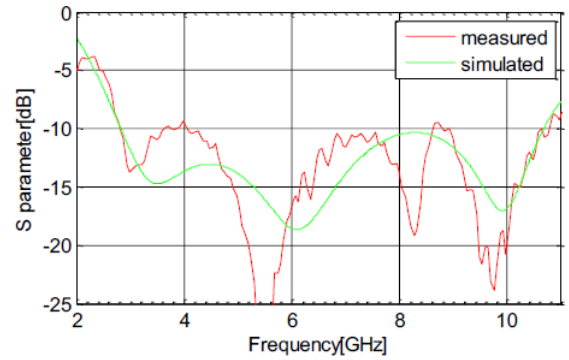

Fig. 2. Simulated and measured reflection coefficient of the capped Bowtie antenna in Fig.1.

array antennas. The working principle of the capped Bowtie antenna can be described as follows. At low frequencies, the radiation is from the bowtie arms, where the cap (much smaller than a half wavelength) works as a capacitor for impedance matching, where the current density on the cap is much lower than the current density on Bowtie arms. At high frequencies, the cap works as a radiating patch while the bowtie arms work as a feeding for the cap, where the current density on the cap is strong. Therefore, at low as well as high frequencies, the capped Bowtie element radiates with almost constant beam. At the middle range of bandwidth, the capped Bowtie geometry makes a Yagi antenna with a reflector by the ground plane, a driven element by the Bowtie arms and a director by the cap whose function is to keep the beam constant.

\section{PCB BASED UWB mMWAVE CAPPED Bowtie ANTENNA}

The capped Bowtie antenna has been extended to millimeter wave regime with PCB (printed circuit board) technology. Fig. 4 shows the design model of a linearly polarized PCB 


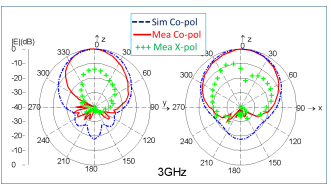

(a)

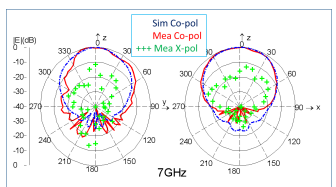

(c) (b)

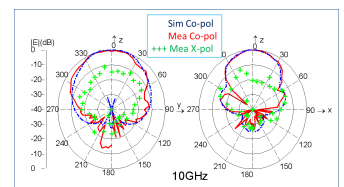

(d)

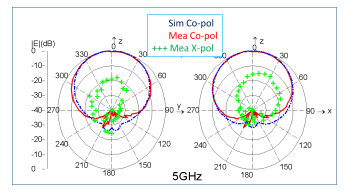

Fig. 3. Simulated and measured radiation patterns of the antenna in Fig.1: (a) $3 \mathrm{GHz},(b(5 \mathrm{GHz}$, (c) $7 \mathrm{GHz}$, and (d) $10 \mathrm{GHz}$.

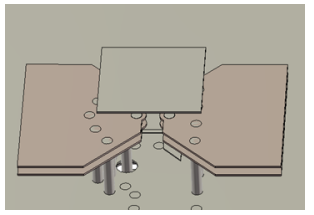

(a)

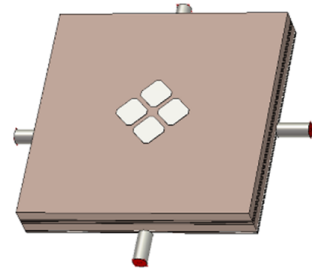

(b)
Fig. 4. PCB based double-layered-arms capped Bowtie with T-feeding for mmWave regime: (a) single linearly polarized element with substrates hidden; (b) $2 \times 2$ dual-polarized array.

based double-layered-arms capped Bowtie with T-feeding and a $2 \times 2$ PCB based dual-polarized capped Bowtie array with the element. With the PCB the manufacture cost is reduced significantly. Fig. 5 shows the simulated results, including reflection coefficient, radiation patterns, radiation efficiency and directivity. From the simulations, it can be observed that

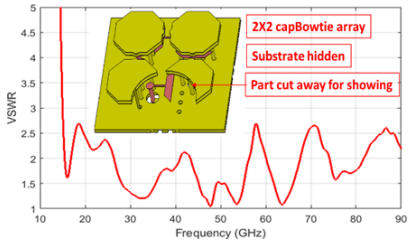

(a)

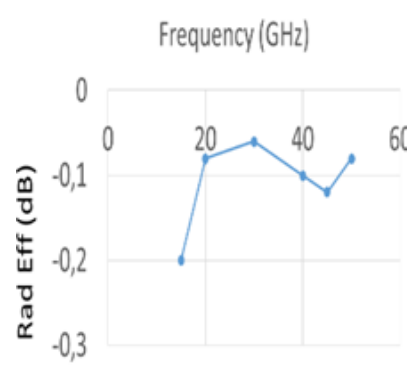

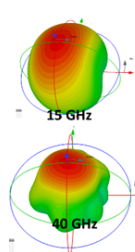

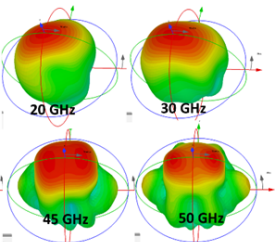

(b)

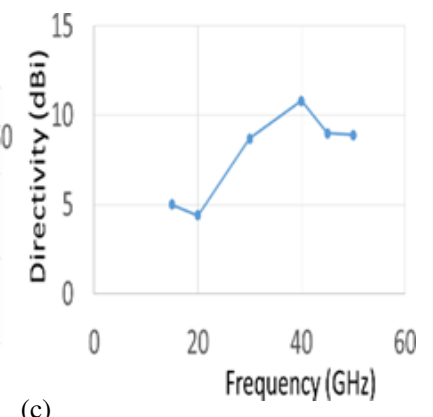

(c)

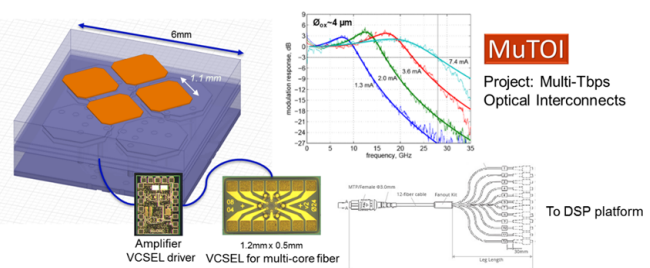

Fig. 6. Aggregation solution by using RF to optical transform to DSP platform.

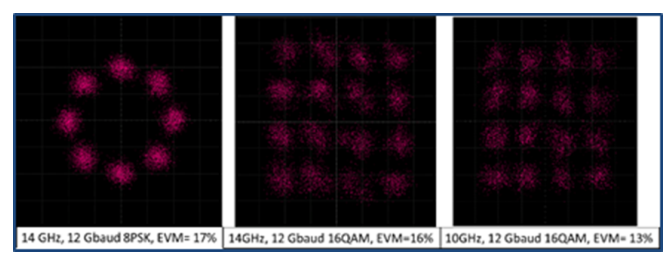

Fig. 7. UWB signal measurement for feasibility with VCSEL fibers.

the antenna has a operation frequency band from $15 \mathrm{GHz}$ to $55 \mathrm{GHz}$.

\section{Aggregation FOR INTEGRATION}

Due to the size of the capped Bowtie array, it is a big challenge to have a good aggregation solution to the integration of the capped Bowtie array with digital signal processing (DSP) platform. A new aggregation solution is proposed and experimentally tested in this paper: Beneath the antenna elements amplifiers with VCSEL (Vertical-Cavity Surface-Emitting Laser) with about $1 \times 1 \mathrm{~mm}^{2}$ in size is used to transform the RF signals to optical signals and then apply six-core optical interconnect fibers to DSP platform, as shown in a detail in Fig. 6. We have done a measurement test, shown in Fig. 7, which has proven the feasibility of this idea.

\section{Conclusions}

An overview on preliminary development of mmWave UWB capped Bowtie antenna array has been presented, and more results will be presented at the conference.

\section{ACKNOWLEDGEMENT}

This work is carried out within the Strategic Innovation Program "Smarter Electronics Systems", a joint venture of Vinnova, Formas and Energy Agency, with a grant no. 201701881.

\section{REFERENCES}

[1] Z. Pi and F. Khan, An introduction to millimeter-wave mobile broadband systems, IEEE Communications Magazine, vol. 49, no. 6, pp. 101107, 2011.

[2] Ericsson source.

[3] Special Issue on Massive MIMO, Journal of communications and networks, vol. 15, no. 4, 2013.

[4] C. Xie, J. Yin, X. Li, F. Pang, Q. Liu, and J. Yang, "An Ultrawideband Dipole With a Director as a Feed for Reflector Antennas", IEEE Antennas and Wireless Propagation Letters, vol. 16, 2017.
Fig. 5. Simulated performance of the antenna in Fig. 4: (a) VSWR; (b) Radiation patterns; (c) Radiation efficiency and directivity. 